\title{
CALIDAD DEL EMPLEO Y SATISFACCIÓN LABORAL EN EMIGRANTES ESPAÑOLES EN PAÍSES EUROPEOS
}

\author{
WORK QUALITY AND JOB SATISFACTION IN SPANISH EMIGRANTS IN EUROPEAN COUNTRIES
}

\author{
Macarena Vallejo-Martín \\ Universidad de Málaga
}

El mercado de trabajo actual se caracteriza por altos índices de desempleo, temporalidad, segmentación y condiciones precarias. La preocupación se vuelve a centrar una vez más en la vertiente numérica del empleo, dejando atrás los esfuerzos por la calidad del mismo. En este contexto la emigración, como era antaño, se vuelve una alternativa posible, y el estudio de la satisfacción laboral por parte de los psicólogos sociales un foco de interés necesario. En este trabajo se vislumbran pistas sobre los factores que pueden determinar la satisfacción laboral, tanto en su dimensión extrínseca como intrínseca, así como los efectos psicosociales que de ella se derivan para esta población. Con ello se pretende ofrecer algunas pautas de intervención con el afán de mejorar la calidad de vida y bienestar de este colectivo.

Palabras claves: Emigración española, Calidad del empleo, Mercado de trabajo, Satisfacción laboral.

The current labour market is characterized by high unemployment rates, temporality, segmentation and precarious conditions. The numerical facet of employment becomes the focus of all concerns once more, casting aside any efforts regarding quality. In this context, emigration becomes a possible alternative once again, as it was in the past, and job satisfaction studies by social psychologists become a necessary focus of interest. This paper outlines the factors that can determine the two dimensions of job satisfaction, both intrinsic and extrinsic ones, and the psychosocial effects for this population that derive from them. With them we intend to offer some intervention guidelines in order to improve quality of life and well-being in this group.

Key words: Spanish emigration, Work quality, labour market, Job satisfaction.

L as migraciones no son un fenómeno del mundo contemporáneo en el que vivimos. A pesar de la gran transcendencia que se le otorga en la actualidad a los flujos migratorios, éstos han existido siempre y son inherentes al ser humano y a las civilizaciones. Sin embargo, en la actualidad el sistema político y económico imperante a nivel mundial ha constituido al planeta como un campo migratorio global, hasta el punto que se hable de la era de las migraciones (Castles y Miller, 1998). Las nuevas tendencias de movilidad humana han transformado el mapa migratorio mundial y la globalización ha participado de manera importante en el desplazamiento, asentamiento y metamorfosis de los perfiles, causas y consecuencias de los movimientos migratorios con respecto a hace cien años (Valero-Matas, Mediavilla, Valero-Otero y Coca, 2015), aunque todo parece indicar, que los comportamientos se reproducen (Valero-Matas, Coca y Miranda-Castañeda, 2010).

La historia de España como un país de emigración tam-

Recibido: 26 junio 2017 - Aceptado: 9 noviembre 2017

Correspondencia: Macarena Vallejo-Martín. Universidad de Má-

laga. Facultad de Psicología. Campus de Teatinos s/n. 29070

Málaga.España.E-mail:mvallejo@uma.es poco es nueva, incluso podría decirse que se trata de un hecho endógeno. La emigración adquirió mucha importancia en el desarrollo económico, cultural y social de España en el pasado y vuelve a tener gran relevancia en el presente. Fenómenos y momentos concretos del siglo XX han marcado recientemente nuestra historia como país emigrante. Ejemplo de ellos pueden ser la dictadura franquista, que durante todo su periodo condenó a miles de personas al exilio, o la modernización de la agricultura que provocó durante la década de los sesenta una fuerte emigración hacia países vecinos derivada del excedente de mano de obra surgido a partir del éxodo de las zonas rurales a las urbanas. La crisis económica acontecida en los últimos años a partir de 2008 caracteriza de nuevo al país como emigrante, aunque continúe siendo receptor de flujos migratorios. A pesar de que esta recesión de la economía pueda enmarcarse en una crisis global, hay factores internos como el freno del "boom inmobiliario" que ha supuesto que se sufra de manera más severa que en otros países y con consecuencias de gran calado. Las cifras sobre las personas que se han desplazado en este último tiempo no están claras, pues existe una discrepancia entre los datos ofrecidos por las instituciones españolas y los aportados por otros países. Estas diferencias, que ya fue- 
ron señaladas por González-Ferrer en 2013, continúan en la actualidad. A modo de ejemplo, podemos citar los casos paradigmáticos de Reino Unido e Irlanda. Para el primero, según el INE (2016) el número de españoles emigrados en 2015 era de 6.038 mientras que los organismos británicos ofrecían una cifra de 50.028 (cinco veces más). En el segundo caso las diferencias se multiplican por ocho, 516 personas según el INE y 4351 según las instituciones irlandesas. Actualmente el INE (2017) señala que el número total de personas españolas que han emigrado desde 2008 al extranjero es de 556.918, de las cuales un $42 \%$ viven en países europeos. Entre los principales destinos se encuentran Reino Unido, Alemania y Francia. Los flujos migratorios han ido incrementándose año a año, aunque se vislumbra una tendencia a la baja a partir de 2016. A continuación, se muestra un gráfico que recoge la evolución desde el año 2008 hacia éstos tres destinos principales

\section{LAS MIGRACIONES DESDE UN ENFOQUE PSICOSOCIAL}

A pesar de la gran relevancia que en la actualidad tiene el fenómeno de la emigración española, son escasos los trabajos a nivel psicosocial referidos a este colectivo (Elgorriaga, Ibabe y Arnoso, 2016; Requés y de Cos, 2003; Vallejo-Martín y Moreno-Jiménez, 2016).

Desde una perspectiva psicológica, la experiencia migratoria se entiende como una etapa transitoria en la que la persona que se desplaza debe hacer frente a un nuevo contexto. Ello conlleva que la persona migrada pase por una fase de ajuste que se conoce como proceso de aculturación (Redfield, Linton y Herskovist, 1936). En él se pueden distinguir dos dimensiones, ajuste sociocultural y ajuste psicológico, que aunque correlacionan entre sí, no

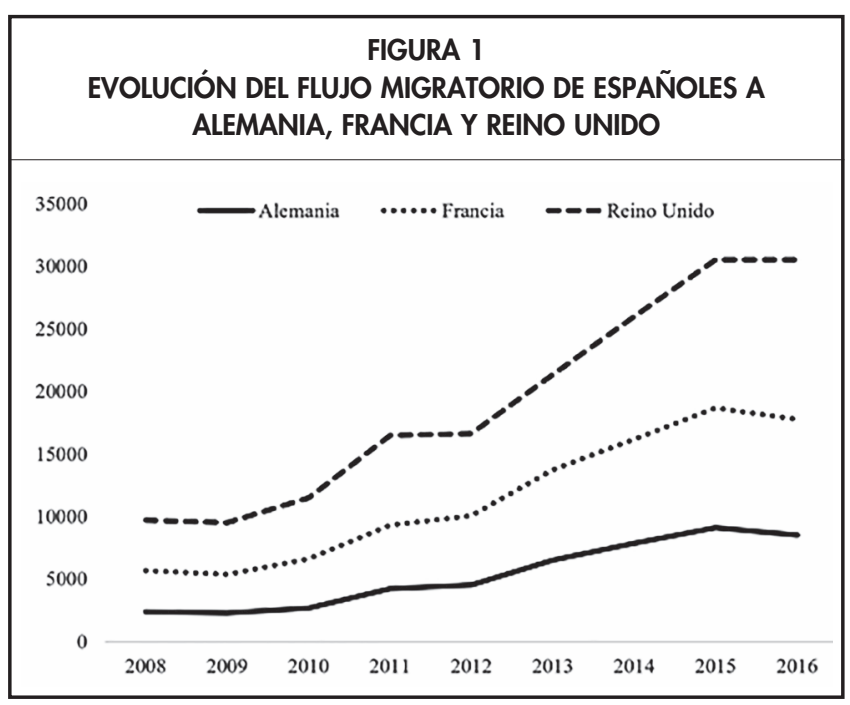

son ajustes idénticos. El primero estaría compuesto por factores como la discriminación percibida (Checa y Arjona, 2005, Piontkowski, Florack, Hoelker y Obdrzálek, 2000; Swawi, 2009) el grado de contacto con la población autóctona (Vallejo, 2009; Ward y Kennedy, 1993) o las dificultades socioeconómicas (De Luca, Bobowik y Basabe, 2011 1). Por su parte, el ajuste psicológico está determinado por variables como el apoyo social (Singh, McBride y Kak, 2015; Vallejo-Martín y Moreno-Jiménez, 2014) o las expectativas migratorias (Berry, 1997; Mähönen, Leinonen y Jasinskaja-Lahti, 2013; Moreno-Jiménez, Ríos-Rodríguez, Canto-Ortiz, San Martín-García y PerlesNova, 2010). Existen varios modelos que explican desde una perspectiva psicosocial los factores que inciden en el proceso de adaptación del proyecto migratorio; entre ellos podemos citar el Modelo de Aculturación (Berry, Kim, Power, Young y Bujaki, 1989), el Modelo de Parker y McEvoy (1993) o el Modelo de Satisfacción Vital para Inmigrantes (Vallejo-Martín y Moreno-Jiménez, 2014), en los que no nos detendremos por exceder las pretensiones de este manuscrito. En cualquier caso, en todos ellos se determina que el empleo y sus condiciones juegan un papel central en la experiencia migratoria y en el ajuste del proceso de aculturación. Tal y como indican varios autores (Becker, 1964; Harris y Todaro, 1970; Piore, 1979; Stark y Bloom, 1985), los flujos migratorios parten de motivaciones económicas, de la racionalidad del mercado de trabajo y de las expectativas profesionales de las personas desplazadas. A pesar de ello, los estudios que relacionan trabajo y ajuste psicológico en población migrante no son abundantes.

\section{LA IMPORTANCIA DEL FACTOR EMPLEO}

Según Aycan y Berry (1996), las personas inmigrantes son especialmente sensibles a los riesgos en el trabajo. Este hecho se produciría por tres razones: 1) las personas inmigrantes tienden a ocupar puestos de unas bajas condiciones laborales, rechazados por autóctonos, con una alta precariedad y bajos salarios (Moreno-Jiménez et al., 2010; Pajares, 2009; Porthé, Amable y Benach, 2007); 2) los inmigrantes tienden a desempeñar funciones por debajo de su cualificación, lo que podría llegar a suponer una ruptura con el contrato psicológico entre trabajador y organización (Morgan y Finniear, 2009) y 3) debido al idioma o pautas culturales diferentes puede producirse una falta de adaptación al puesto de trabajo aumentando el estrés aculturativo (Ramos-Villagrasa, García-Izquierdo y García-Izquierdo; 2011).

El trabajo debería ser una fuente de realización personal, seguridad, contacto social y salud física y mental (Gamero, 2009; Salanova, Gracia y Peiró, 1996). Sin 
embargo, la complejidad del mercado de trabajo $y$, en muchos casos, los altos índices de precariedad están afectando al bienestar y a la salud de la población trabajadora con amplias consecuencias en la economía global (European Agency for Safety and Health at Work, 2009). Estas repercusiones son aún más acuciantes para el trabajador inmigrante, que presenta peores condiciones laborales, tiene que adaptarse a nuevas formas de organización (Ramos-Villagrasa y García-Izquierdo, 2007) y debe realizar los esfuerzos propios del proceso de aculturación (Bhattacharya y Schoppelrey, 2004; Kosic, 2004; Vallejo, 2009).

Las dificultades y consecuencias negativas debido a las condiciones del empleo no le son ajenas a la emigración española. A continuación, se recogen ejemplos de cómo algunos autores reflejan esta realidad referida a la emigración española de la década de los sesenta:

"La discriminación laboral fue otro elemento negativo para la integración de los emigrantes españoles. Las diferencias salariales con los nativos, las casi inexistentes relaciones laborales, las restricciones de acceso a los mismos beneficios de los oriundos, la falta de preparación y la dificultad con el idioma no proporcionaron una equiparación formativa con los nativos y, por ende, resultó prácticamente inexistente la movilidad laboral" (Valero-Matas, Mediavilla, Valero-Otero y Coca, 2015).

"los emigrantes españoles arrojan siempre el mínimo de capacitación profesional, ocupan los puestos de inferior categoría dentro de la escala profesional europea; la mayor parte están empleados en la industria, pero no como obreros especializados, sino en condición de peones 0 en puestos que requieren una baja cualificación o un rápido aprendizaje" (Meseguer, 1975, p. 412).

Sin embargo, el perfil del trabajador español emigrante en países europeos cambió considerablemente con el inicio de la democracia y la entrada en la Comunidad Económica Europea. Su carácter era menos masivo y estaba compuesto por trabajadores de multinacionales, investigadores 0 aventureros en búsqueda de nuevas oportunidades empresariales o vitales. Además, destacaban los perfiles de profesionales cada vez más cualificados en búsqueda de reconocimiento social y laboral. Surgen en ese momento nuevas tipologías de emigrantes tanto en Europa como en el contexto español que van más allá de las migraciones laborales. Se corresponden con nuevas formas de movilidad, donde los elementos económicos aparecen combinados con otras motivaciones como son el tiempo libre o las oportunidades de nuevos estilos con calidad de vida (Alaminos, Albert y Santacreu, 2010). En concreto, para esos años, la emigración española experimenta una movilidad social as- cendente en la clase social en comparación al estatus antes del desplazamiento y respecto a otras migraciones intraeuropeas (Recchi, 2006).

Pero ¿̇cómo es la relación actual entre empleo y emigración española en los países europeos?, ¿están satisfechas en su trabajo las personas desplazadas? La crisis económica iniciada en 2008 vuelve a cambiar el panorama migratorio español y con ello también las relaciones con países vecinos y las características de la emigración. El contexto socioeconómico en nuestro país se define por unos altos índices de desempleo, precariedad laboral y social, falta de oportunidades y jóvenes altamente cualificados que visualizan la emigración como única alternativa laboral. Los efectos de este panorama son a corto, medio y, probablemente, largo plazo, tanto a nivel colectivo como individual. Tal y como indica Santos (2016), el fenómeno de la fuga de cerebros se ha convertido en el periodo de crisis en un icono, del cual es difícil determinar con exactitud su volumen numérico, pero del que es indiscutible su magnitud simbólica. Las autoras Moldes y Gómez (2015) en su libro ¿ᄅor qué te vas? Jóvenes españoles en Alemania determinan que España se encuentra en su tercera fase migratoria, en la que se nos devuelve de nuevo a la casilla de salida. La obra también recoge una descripción del éxodo juvenil compuesto por personas de una alta cualificación y con un alto manejo de las nuevas tecnologías, cuya principal razón para emigrar ha sido la situación agónica del mercado de trabajo español y cuya satisfacción laboral en destino está determinada por algunos factores como el conocimiento de la lengua. Así, se esbozan historias de éxito profesional junto a otras de condiciones de alta precariedad y sobrecualificación con agravios comparativos importantes respecto a la población autóctona alemana.

\section{CALIDAD EN EL EMPLEO Y SATISFACCIÓN LABORAL}

Como se describía anteriormente, el actual mercado de trabajo está caracterizado por la individualización, segmentación, flexibilización, precarización, etc. Por ello, hablar de la calidad en el empleo en tiempos de crisis socioeconómica no es una cuestión baladí, y menos aún, refiriéndonos a la población migrante.

En los últimos tiempos se ha acentuado de nuevo la polarización y tensión entre cantidad y calidad del trabajo. Esta disyuntiva tiene un importante recorrido y se ha ido consolidando como un creciente campo de análisis de la investigación económica, social y psicológica (González y Guillén, 2009; Narocki, Zimmermann, Artazcoz, Gimeno y Benavides, 2009; Sola, 2011). De esta forma, aunque parecía que la calidad en el empleo se colocaba en el centro del tablero de las políticas públicas, la crisis 
y los altos índices de desempleo vuelven a otorgar la supremacía a las cuestiones vinculadas con la cantidad. Sin embargo, hay suficientes datos que corroboran que la calidad en el empleo aumenta las capacidades de los trabajadores, mejora las condiciones de trabajo, aumenta la productividad, fomenta el bienestar social, y en último término, mejora la salud física, psíquica y emocional de la persona empleada (Cottinni y Lucifora, 2013; Dahl, Nesheim y Olsen, 2009; Davoine, Erhel y Guergota-Lariviere, 2009; Green et al., 2013).

Tradicionalmente la calidad en el empleo se ha movido en dos dimensiones que, aunque a priori pueden parecer opuestas, son complementarias para un abordaje integral. Se tratan de la dimensión objetiva, más relacionada con las condiciones físicas, seguridad en el trabajo, procesos organizativos y de gestión, y la dimensión subjetiva, que englobaría cuestiones como la satisfacción laboral, el compromiso con la organización, motivación, etc. Es decir, la dimensión objetiva hace alusión al entorno laboral, y la subjetiva, a las personas empleadas. Estas dos dimensiones no deben ser enemigas, sino aliadas necesarias. Así, la calidad en el empleo debe entenderse como un concepto multidimensional que debe ser abordado desde diferentes perspectivas (micro y macro económica, psicológica y sociológica) y para distintos actores (personas empleadas, organizaciones y puestos de trabajo). Acogemos, por ello, la definición de calidad laboral aportada por los autores Díaz-Chao, Ficapal-Cusí y Torrent-Sellens (2015) por ser una de las más completas: "la calidad del empleo es un estado general de satisfacción que incluye aspectos objetivos de bienestar material, relaciones satisfactorias con el entorno físico y social, y salud objetivamente percibida; y aspectos subjetivos de bienestar físico, psicológico y social". También es importante señalar las distintas dimensiones que recoge la Comisión Europea (2008) para medir la calidad del empleo en sus países: 1) calidad intrínseca del trabajo, sus habilidades y competencias; 2) aprendizaje y desarrollo de la carrera profesional; 3) igualdad de género; 4) salud y seguridad en el trabajo; 5) flexibilidad y seguridad del empleo; 6) inclusión y acceso a los mercados de trabajo; 7) organización del trabajo y conciliación familiar; 8) diálogo social y participación; 9) diversidad y no discriminación y 10) resultado económico agregado y productividad. Como vemos se trata de un concepto amplio y del que es complejo su medición.

Desde un abordaje psicológico, la calidad en el empleo está íntimamente relacionada con la satisfacción laboral (Green, 2010; González, Peiró y Bravo, 1996; May y Lau, 1988; Sirgy, 2001). Locke (1976) la concibe como un estado emocional positivo o placentero de las experiencias laborales del trabajador. Las características del propio trabajo y cómo son percibidas y valoradas por la persona empleada condicionarán esa respuesta afectiva. Para Spector (1997), la satisfacción laboral es simplemente el sentimiento de las personas hacia su trabajo, basado en la valoración de aspectos satisfactorios e insatisfactorios del mismo. Por su parte, Fabra y Camisón (2009) consideran que la satisfacción laboral es una valoración conjunta de las ganancias monetarias y no monetarias que les reportan sus trabajos, de acuerdo con sus preferencias personales y sus expectativas.

Aunque se trata de un concepto difuso, en la actualidad las investigaciones parten del carácter bidimensional aportado por Warr, Cook y Wall (1979) distinguiendo entre satisfacción laboral extrínseca e intrínseca. La primera se centra en la satisfacción del trabajador con aspectos relativos a la organización del trabajo, como la jornada laboral, la remuneración o las condiciones físicas del mismo. Por su parte, la satisfacción laboral intrínseca aborda aspectos como el reconocimiento obtenido por el trabajo, responsabilidad, promoción y cuestiones relativas al contenido de la tarea.

En el siguiente apartado se describen los resultados de un estudio con el que pretendemos esbozar un análisis sobre la satisfacción laboral para el colectivo de emigrantes españoles en países europeos. Puesto que la mayoría de estas personas se desplazan por motivos relacionados con el empleo, cómo valoran el mismo y qué grado de satisfacción les reporta puede ser una cuestión de singular importancia en su proyecto migratorio.

\section{SATISFACCIÓN LABORAL EN EMIGRANTES ESPAÑOLES}

El estudio al que hacemos referencia está compuesto por personas españolas (un total de 816 ) que han emigrado relativamente hace poco tiempo (periodo medio de veintiún meses) a países europeos (fundamentalmente a Reino Unido, Alemania y Francia). Nos encontramos con un perfil de personas jóvenes (de una media de treinta años), con mayoría de mujeres, cuyas principales motivaciones para emigrar han sido la búsqueda de empleo y ejercer una profesión acorde al nivel de cualificación. Casi dos tercios poseen estudios universitarios. La tasa de empleo roza casi el $90 \%$, aunque algo más de la mitad lo hacen en funciones por debajo de su nivel formativo.

La primera conclusión que podemos extraer del estudio es que se confirman las dos dimensiones de la satisfacción laboral. Según los datos, la población española residente en países europeos tiene unos niveles medios de satisfacción en el trabajo, aunque son mayores en su dimensión extrínseca que en la intrínseca. Igualmente, los factores 
con los que se asocia cada una de ellas son diferentes en cada caso, aunque encontramos algunos comunes.

Los resultados muestran mayores niveles de satisfacción (extrínseca e intrínseca) para aquellas personas que tienen un contrato indefinido, a tiempo completo, con mayores ingresos y ajustado a su nivel formativo. Por otro lado, obtenemos una mayor satisfacción laboral extrínseca en personas con estudios universitarios respecto a aquellas de estudios primarios o secundarios. Por su parte, para la satisfacción laboral intrínseca también son importantes cuestiones como el tipo de tareas, en el sentido de que éstas sean estimulantes y no repetitivas o aburridas, no percibir un control estricto por parte de los superiores o no tener un horario muy extenso. En cualquier caso, los resultados ponen de manifiesto que las condiciones laborales determinan ambos tipos de satisfacción (Tabla 1).

Un hallazgo relevante es que, al contrario que en otras investigaciones en las que se indican que las personas inmigrantes se insertan al principio en trabajos precarios de baja remuneración pero que tienden a ir mejorando con el tiempo (Martínez, 1996; Patiño y Kirchner,

\begin{tabular}{|c|c|c|c|c|}
\hline \multicolumn{5}{|c|}{$\begin{array}{c}\text { TABLA } 1 \\
\text { DIFERENCIAS EN S. L. EXTRÍNSECA Y S. L. INTRÍNSECA SEGÚN } \\
\text { SEXO, NIVEL DE ESTUDIOS, CONDICIONES LABORALES Y } \\
\text { TIEMPO DE RESIDENCIA (T-STUDENT Y ANOVA) }\end{array}$} \\
\hline & $\begin{array}{l}\text { atisfacción laboral } \\
\text { extrínseca (1-7) }\end{array}$ & $p$ & $\begin{array}{c}\text { Satisfacción laboral } \\
\text { intrínseca }(1-7)\end{array}$ & $p$ \\
\hline General & 4.94 & & 4.72 & \\
\hline Sexo & & 628 & & .302 \\
\hline Hombres & 4.97 & & 4.80 & \\
\hline Mujeres & 4.92 & & 4.67 & \\
\hline Nivel de estudios & & .042 & & .123 \\
\hline Primarios & 5.02 & & 4.72 & \\
\hline Secundarios & 4.95 & & 4.77 & \\
\hline Universitarios & 5.26 & & 4.78 & \\
\hline Tipo de contrato & & .000 & & .000 \\
\hline Temporal & 4.64 & & 4.41 & \\
\hline Servicio/Proyecto & 4.46 & & 4.28 & \\
\hline Indefinido & 5.21 & & 4.99 & \\
\hline Tipo de jornada & & .000 & & .000 \\
\hline Completa & 5.09 & & 4.92 & \\
\hline Parcial & 4.55 & & 4.19 & \\
\hline Ajuste al puesto & & .000 & & .000 \\
\hline Cualificación adecuada & 5.34 & & 5.36 & \\
\hline Sobrecualificación & 4.42 & & 3.86 & \\
\hline Ingresos & & .000 & & .000 \\
\hline$<1000$ & 4.31 & & 3.99 & \\
\hline $1000-2500$ & 5.06 & & 4.75 & \\
\hline$>2500$ & 5.56 & & 5.59 & \\
\hline Tiempo de residencia & & 1.29 & & .009 \\
\hline$<6$ meses & 4.74 & & 4.43 & \\
\hline 6 meses - 1 año & 4.96 & & 4.74 & \\
\hline $1-5$ años & 5.01 & & 4.80 & \\
\hline$>5$ años & 5.14 & & 5.22 & \\
\hline
\end{tabular}

2009), en nuestro estudio la satisfacción laboral extrínseca no presenta diferencias significativas en función del tiempo de residencia. Sin embargo, las personas sí muestran mayores niveles de satisfacción laboral intrínseca con el paso de los años y ésta parece ser una cuestión determinante. Además, encontramos otras dos cuestiones de interés. La primera, que el conocimiento del idioma es un factor clave en relación a la satisfacción laboral en sus dos dimensiones. La segunda, que no se detectan diferencias entre hombres y mujeres ni en la satisfacción laboral extrínseca ni en la intrínseca.

Respecto a la relación de la satisfacción laboral con otras variables psicosociales, los resultados arrojan que tanto la satisfacción laboral extrínseca como la intrínseca están relacionadas con la satisfacción vital y el ajuste psicológico de las personas desplazadas. La satisfacción vital en población migrada se corresponde a la valoración que hace el sujeto de su propia experiencia migratoria en relación a que su vida se desarrolle de acuerdo a metas, creencias, valores y deseos propios (Marrero y Carballeira, 2010) y está muy relacionada con las expectativas puestas en el proceso migratorio. Los resultados encontrados estarían acordes a otros estudios recientes sobre emigración española y calidad en el empleo (Elorriaga, Ibabe y Arnoso, 2016; Vallejo-Martín y Moreno Jiménez, 2016), que destacan como la satisfacción laboral y unas buenas condiciones laborales tienen una correlación positiva con la satisfacción con la vida y en sentido inverso con el estrés percibido. Asimismo, otro estudio de la misma índole pone de manifiesto que unas condiciones laborales negativas constituyen un factor predictivo para padecer el síndrome de burnout en este colectivo (Vallejo-Martín, 2017).

Por último, es importante señalar que los datos también muestran que la satisfacción laboral en sus dos dimensiones está relacionada con una mejor autoestima, mayor percepción de apoyo social en el nuevo lugar de residencia y menor prejuicio percibido en el ámbito laboral por parte de la población autóctona (Tabla 2).

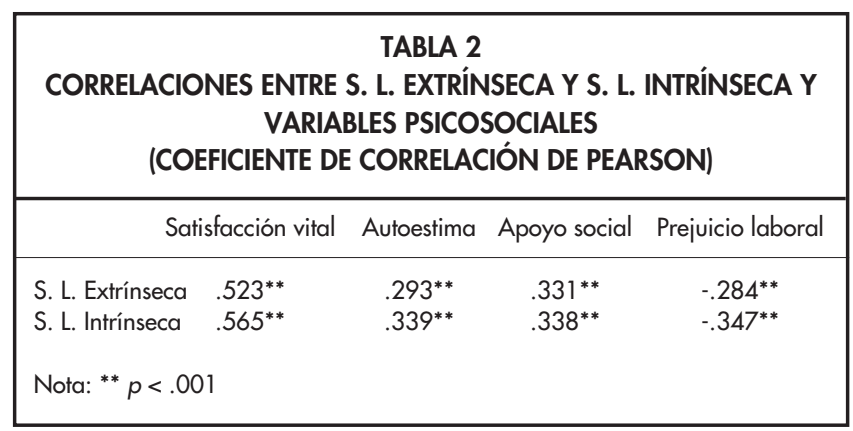




\section{CONCLUSIONES}

Como se ha expuesto a lo largo de este trabajo, España vive en la actualidad un éxodo de personas, principalmente jóvenes, que ven la emigración como alternativa plausible a un mercado de trabajo precario, sin oportunidades y con pocas perspectivas de mejora. Los flujos a países vecinos nos recuerdan a épocas de antaño, en la que muchos de nuestros abuelos y abuelas tuvieron que desplazarse como consecuencia de la situación política y económica del momento. Aunque mucho ha cambiado nuestro país en los últimos tiempos, el "refugio" en Alemania, Francia o Reino Unido vuelve a estar de actualidad. Sin embargo, hay características definitorias para esta nueva oleada de emigración. En primer lugar, el perfil de las personas desplazadas, de una alta cualificación y formación, que da origen a la denominación "fuga de cerebros". La segunda, el uso y acceso a las nuevas tecnologías por parte de los que emigran, lo que facilita las redes de información y el contacto con familiares y amigos en origen. Por último, y no menos importante, la pertenencia a la Unión Europea, lo que elimina los requisitos de acceso y permanencia a otros países bajo el amparo de la ciudadanía comunitaria.

Según los participantes de nuestro estudio, el principal motivo de la emigración es encontrar un empleo y ejercer una profesión acorde al nivel formativo, y es esta última cuestión la que más parece incidir en la satisfacción laboral. Las características objetivas y condiciones laborales como el tipo de jornada, el tipo de contrato o el nivel de ingresos también son importantes para determinar la satisfacción en sus dos dimensiones. Es decir, podríamos establecer una relación entre la calidad de las condiciones del trabajo y la satisfacción laboral. Sin embargo, las personas señalan como factores principales para estar satisfechos trabajar en la propia profesión, sentirse reconocidos y valorados, desarrollar unas tareas que permitan el desempeño de sus capacidades o tener libertad parar ejercer determinadas funciones. $\mathrm{Pa}$ rece ser que los emigrantes españoles no sólo buscan un salario que les permita la supervivencia o mantenerse en el país de acogida, sino también un reconocimiento social y laboral del que carecen en España. El cumplimento de estas expectativas, en gran medida, va a determinar el curso del proyecto migratorio, pudiendo establecerse un vínculo entre satisfacción laboral y otras variables psicosociales. Así, la satisfacción laboral se relaciona con hacer un juicio global positivo de la vida, tener un mejor concepto de sí mismo, percibir menos discriminación por parte de la sociedad de acogida en el terreno laboral y disfrutar de más redes disponibles en el nuevo país de destino.
Como conclusión, podemos dilucidar que la calidad y las características del empleo y el grado de satisfacción con el mismo son una piedra angular en el proyecto migratorio de las personas emigrantes españolas, con implicaciones en su ajuste aculturativo y su satisfacción con la vida y con hondas implicaciones psicosociales. A España le quedan aún muchos retos por delante, en los que la disyuntiva entre cantidad y calidad del empleo debe estar superada y en los que se otorgue a la generación más preparada de la historia de nuestro país el reconocimiento laboral que le corresponde.

\section{CONFLICTO DE INTERESES}

No existe conflicto de intereses.

\section{REFERENCIAS}

Alaminos, A. Albert, M. C. \& Santacreu, O. (2010). La movilidad social de los emigrantes españoles en Europa. Revista Española de Investigaciones Sociológicas, 129, 13-35.

Aycan, Z. \& Berry, J. W. (1996). Impact of employment related experiences on immigrants' psychological wellbeing and adaptation to Canada. Canadian Journal of Behavioural Science, 28, 240-251.

Bhattacharya, G., \& Schoppelrey, S. L. (2004). Pre-immigration beliefs of life success, postimmigration experiences, and acculturative stress: South Asian immigrants in the United States. Journal of Immigrant Health, 6, 83-92.

Becker, G. S. (1964). Human Capital: A theoretical and empirical analysis with special reference to education. New York: National Bureau of Economic Research.

Berry, J. W. (1997). Immigration, acculturation and adaptation. Applied Psychology: An International Review, 46, 5-68. doi: 10.1111/j.14640597.1997.tb01087.x

Berry, J. W., Kim, U., Power, S., Young, M. \& Bujaki, M. (1989). Acculturation attitudes in plural societies. Applied Psychology, 38, 185-206.

Castles, S. \& Miller, M. J (1998). The age of migration: International population movements in the modern world. London: Macmillan.

Checa, J. C. \& Arjona, Á. (2005). El vecino no deseado. Situación residencial de los inmigrantes africanos en Almería (España). Revue Européenne des Migrations Internationales, 21 (3), 179-208.

Comisión Europea (2008). Measuring the quelity of employment in the EU. En Comisión Europea, Employment in Europe report 2008 (pp. 147-165). Luxenburgo: Autor.

Cottini, E. \& Lucifora, C. (2013). Mental health and wor- 
king conditions in Europe. Industrial and Labour Relations Review, 66, 958-988.

Dahl, S. A., Nesheim, T. \& Olsen, K. M. (2009). Quality of work: Concept and measurement. En A. M. Guillén \& S. A. Dahl (Eds.), Quality of work in the European Union: Concept, data and debates from a transnational perspective (pp. 19-40). Bruselas: Peter Lang.

De Luca, S., Bobowik, M., \& Basabe, N. (2011). Adaptación sociocultural de inmigrantes brasileños en el País Vasco: Bienestar y aculturación. Revista de Psicología Social, 26, 275-294. doi:10.1174/021347411795448983

Díaz-Chao, A., Ficapal-Cusí, P., \& Torrent-Sellens, J. (2015). Determinantes multidimensionales en la calidad percibida del empleo. Evidencia empírica para España. Revista Internacional de Sociología, 73(1), 114. doi:10.3989/ris.2013.03.25

Elorriaga, E., Ibabe, I. \& Arnoso, A. (2016). Españoles que emigran a países de la Unión Europea: predictores de su ajuste psicológico. Revista de Psicología Social, 31(2), 317-351. doi: 10.1080/02134748.2016.1143178

European Agency for Safety and Health at Work: Milczarek, M., Schneider, E., Rial Gonzalez, E. (2009). $\mathrm{OSH}$ in figures: stress at work-facts and figures. Luxembourg: European Communities.

Fabra, M. E. \& Camisón, C. (2009). Direct and Indirect effects of education on job satisfaction: A structural equation model for the Spanish case. Economics of Education Review, 28, 600-610.

Gamero, C. (2009). Valoración de las características de los empleos por nativos e inmigrantes: el enfoque de la satisfacción con la vida. Principios. Estudios de Economía Política, 14, 93-111.

González, S. \& Guillén, A. M. (2009). La calidad del empleo en la Unión Europea. Debate político y construcción de indicadores. Revista del Ministerio de Trabajo e Inmigración, 81, 71-88.

González, P., Peiró, J. M. \& Bravo, M. J. (1996). Calidad de vida laboral. En J.M. Peiró \& F. Prieto (Eds.), Tratado de Psicología del Trabajo. Aspectos psicosociales del trabajo, (vol. 2, pp. 161-185). Madrid: Síntesis.

González-Ferrer, A. (2013). La nueva emigración española. Lo que sabemos y lo que no. Zoom Político. Madrid, Laboratorio de Alternativas.

Green, F. (2010). Well-being, Job Satisfaction and Labour Mobility. Labour Economics, 17, 897-903. doi: 10.1016/i.labeco.2010.04.002

Green, F., Mostafa, T., Parent, A., Vermeylen, G., Van Houten, G., Biletta, I. \& Lyly-Yrianainen, M. (2013). Is job quality becoming more unequal? Industrial and
Labor Relations Review, 66, 753-784.

Harris, J. R. \& Todaro, M. P. (1970). Migration, unemployment and development: A two-sector analysis. American Economic Review, 60, 126-142.

INE (2017). Estadística de migraciones. Resultados anuales: Flujo de emigración con destino al extranjero por año, país de destino y nacionalidad. http://www.ine.es

Kosic, A. (2004). Acculturation strategies, coping process and acculturative stress. Scandinavian Journal of Psychology, 45, 269-278. doi: 10.1111/j.14679450.2004.00405.x

Locke, E. A. (1976). The nature and causes of job satisfaction. En M.D. Dunnette (Ed.), Handbook of Industrial and Organizational Psychology, (pp. 1297-1349). Chicago, IL: Rand McNally.

Mähönen, T. A., Leinonen, E., \& Jasinskaja-Lahti, I. (2013). Met expectations and the wellbeing of diaspora immigrants: A longitudinal study. International Journal of Psychology, 48, 324-333. doi:10.1080/00207594.2012.662278

Marrero, R. J. \& Carballeira, M. (2010). El papel del optimismo y del apoyo social en el bienestar subjetivo. Salud Mental, 33, 39-46.

May, B. E. \& Lau, R. S. M. (1988). A longitudinal study of quality of work life and business performance. South Dakota Business Review, December.

Martínez, M. C. (1996). Análisis psicosocial del prejuicio. Madrid: Síntesis.

Meseguer, J. (1975). La emigración española en los países de la CEE. Revista de Instituciones Europeas, 2, 405-421.

Moldes, R. \& Gómez, F. (2015). ¿Por qué te vas? Jóvenes españoles en Alemania. Madrid: Los Libros de la Catarata.

Moreno-Jiménez, M. P, Ríos-Rodríguez, M. L., Canto-Ortiz, J., San Martín-García, J. \& Perles-Nova, F. (2010). Satisfacción laboral y burnout en trabajos pocos cualificados: diferencias entre sexos en población inmigrante. Revista de Psicología del Trabajo y de las Organizaciones, 26, 255-265.

Morgan, A., \& Finniear, J. (2009). Migrant workers and the changing psychological contract. Journal of European Industrial Training, 33, 305-322. doi: 10.1108/03090590910959272

Narocki, C., Zimmermann, M., Artazcoz, L., Gimeno, D. \& Benavides, F. (2009). Encuestas de condiciones de trabajo y salud en España: comparación de los contenidos del cuestionario del trabajador. Archivo de Prevención de Riesgos Laborales, 12, 60-68.

Pajares, M. (2009). Inmigración y mercado de trabajo. Informe 2009. Madrid: Documentos del Observatorio 
Permanente de la Inmigración. Ministerio de Trabajo e Inmigración: Gobierno de España.

Parker, B. \& McEvoy, G. M. (1993). initial examination of a model of intercultural adjustment. International Journal of Intercultural Relations, 17, 355-379.

Patiño, C. y Kirchner, T. (2009). Estrés y calidad de vida en inmigrantes latinoamericanos en Barcelona. Ansiedad y Estrés, 15, 49-66.

Piontkowski, U., Florack, A., Hoelker, P. \& Obdrzálek, P (2000). Predicting acculturation attitudes of dominat and no-dominant groups. International Journal of Intercultural Relations, 24, 1-26.

Piore, M. J. (1979). Birds of passage. Migrant labor and industrial societies. London: Cambridge University Press.

Porthé, V., Amable, M. \& Benach. J. (2007). La precariedad laboral y la salud de los inmigrantes en España: ¿̇qué sabemos y qué deberíamos saber? Archivos de Prevención Riesgos Laborales, 10, 34-39.

Ramos-Villagrasa, P. J. \& García-Izquierdo, A. L. (2007). La medida del síndrome de Ulises. Ansiedad y Estrés, 13, 253-268.

Ramos-Villagrosa, P., García-Izquierdo, A. L. \& GarcíaIzquierdo, M. (2011). Acculturative strategies and emotional exhaustion differences between immigrant and national workers in Spain. Ansiedad y Estrés, $17(1), 63-74$.

Recchi, E. (2006). Spatial mobility and social mobility in the EU. Firenze: Pioneur Final Conference.

Redfield, R., Linton, R. \& Herskovist, M. J. (1936). Memorandum on the study of acculturation. American Anthropologist, 38, 149-152.

Reques, P. \& Cos, O. (2003). La emigración olvidada: la diáspora española en la actualidad. Papeles de Geografía, 37, 199-216.

Salanova, M., Gracia, F. J. \& Peiró, J. M. (1996). Significado del trabajo y valores laborales. En J. M. Peiró y F. Prieto (Eds.), Tratado de Psicología del Trabajo. Vol. II: Aspectos psicosociales del trabajo, (pp. 3563). Madrid: Síntesis

Santos, A. (2016). Reseña literaria: ¿Por qué te vas? Jóvenes españoles en Alemania. Revista Española de Investigaciones Sociológicas, 155, 161-165.

Singh, S., McBride, K., \& Kak, V. (2015). Role of social support in examining acculturative stress and psychological distress among Asian American immigrants and three sub-groups: Results from NLAAS. Journal of Immigrant and Minority Health, 17, 1597-1606. doi:10.1007/s10903-015-0213-1

Sirgy, M. J. (2001). Handbook of quality-of-Life research: An ethical marketing perspective. Berlin. Sprin-
ger-Verlag. doi: 10.1007/978-94-015-9837-8

Sola, J. (2011). La calidad del empleo en España: una aproximación teórica y empírica. Política y Sociedad, 48, 411-415.

Spector, P. E. (1997). Job satisfaction: Application, assessment, causes, and consequences. London: Sage.

Stark, O. \& Bloom, D. E. (1985). The new economics of labor migration. The American Economic Review, 75, 173-178.

Swami, V. (2009). Predictors of sociocultural adjustment among sojourning Malaysian students in Britain. International Journal of Psychology, 44, 266-273. doi:10.1080/00207590801888745

Valero-Matas, J. A., Mediavilla, J. J, Valero-Oteo, I. \& Coca, J. (2015). El pasado vuelve a marcar el presente: la emigración española. Papeles de Población, 21(83), 41-74.

Valero-Matas, J. A., Coca, J. R \& Miranda-Castañeda, S. (2010). The migratory flows in Spain: an analysis of the migration and immigration input from European Union. Papeles de Población, 16(65), 233- 256.

Vallejo-Martín, M. (2009). Satisfacción vital en el proceso de aculturación de inmigrantes: propuesta de un modelo. [Tesis Doctoral no publicada], Departamento de Psicología Social, Antropología Social, Trabajo Social y Servicios Sociales, Universidad de Málaga.

Vallejo-Martín, M. (2017). Una aproximación al síndrome de burnout y las características laborales de emigrantes españoles en países europeos. Journal of Work and Organizational Psychology, 33, 137-15. doi:10.1016/j.rpto.2017.02.001

Vallejo-Martín, M. \& Moreno-Jiménez, M. P. (2014). Del culturalismo al bienestar psicológico: propuesta de un modelo de satisfacción vital en el proceso de aculturación de Inmigrantes. Boletín de Psicología, 110, 53-67.

Vallejo-Martín, M. \& Moreno-Jiménez, M.P. (2016). Satisfacción vital y su relación con otras variables psicosociales en población española residente en Alemania. Escritos de Psicología, 9(2), 12- 21.

Ward, C., \& Kennedy, A. (1993). Psychological and sociocultural adjustment during crosscultural transitions: A comparison of secondary students overseas and at home. International Journal of Psychology, 28, 129147. doi:10.1080/00207599308247181

Warr, P., Cook, J. \& Wall, T. (1979). Scales for the measurement of some work attitudes and aspects of psychological well-being. Journal of Occupational Psychology, 52, 129-148. doi:10.1111/i.20448325.1979.tb00448.x 